\title{
Factores de riesgo de preeclampsia identificables durante la primera consulta del control prenatal
}

\begin{abstract}
Objetivo
Determinar el riesgo de preeclampsia según factores de riesgo que pueden estar presentes en la primera consulta prenatal.

\section{Fuente y selección de datos}

Los autores realizaron la búsqueda sobre factores de riesgo prenatales identificables durante la primera consulta prenatal y el desarrollo subsecuente de preeclampsia. Se revisó MEDLINE (desde 1966 hasta 2002) y EMBASE (desde 1974 hasta 2002). Se seleccionaron estudios retrospectivos y prospectivos (de cohorte y de caso-control) que cumplieran los criterios de inclusión basados en una evaluación de su calidad. Se realizó una revisión sistemática de los estudios seleccionados calculándose los riesgos relativos no ajustados para cada factor de riesgo analizado.
\end{abstract}

\section{Resultados}

Se incluyeron 52 estudios (13 prospectivos de cohorte, 25 retrospectivos de cohorte y 14 estudios de caso-control) para la revisión sistemática, cuyos resultados se resumen en el cuadro 1.
Cuadro 1: factores de riesgo de preeclampsia.

\begin{tabular}{|c|c|c|c|}
\hline & Factor de riesgo & Riesgo relativo & IC95\% \\
\hline \multirow{3}{*}{$\begin{array}{l}\text { Anteriores al } \\
\text { embarazo }\end{array}$} & Pre-eclampsia previa & 7,19 & $5,85-8,83$ \\
\hline & Anticuerpos antifosfolípidos & 9,72 & $4,34-21,75$ \\
\hline & Diabetes pre-gestacional & 3,56 & $2,54-4,99$ \\
\hline Embarazo múltiple & & 2,93 & $2,04-4,21$ \\
\hline Nuli paridad & & 2,91 & $1,28-6,61$ \\
\hline \multicolumn{2}{|c|}{ Antecedente familiar de preeclampsia } & 2,9 & $1,70-4,93$ \\
\hline \multicolumn{2}{|c|}{ Más de $80 \mathrm{mmHg}$ de tensión arterial diastólica } & 1,38 & $1,01-1,87$ \\
\hline \multirow[t]{2}{*}{ Obesidad } & Antes del embarazo & 2,47 & $1,66-3,67$ \\
\hline & En la primera consulta & 1,55 & $1,28-1,88$ \\
\hline \multicolumn{2}{|c|}{ Edad materna mayor a 40 años en multíparas } & 1,96 & $1,34-2,87$ \\
\hline
\end{tabular}

Algunos estudios individuales encontraron el riesgo elevado en mujeres con intervalo intergenésico de más de diez años, enfermedades autoinmunes, enfermedad renal e hipertensión crónica.

\section{Conclusión}

Estos factores pueden ser empleados para evaluar riesgo de preeclampsia en la primera consulta prenatal y planificar una vigilancia adecuada para el resto del embarazo.

Fuente de financiamiento: algunas fotocopias fueron donadas por "Action on Pre-eclampsia".

\section{Comentario}

La preeclampsia constituye una de las principales causas de morbi-mortalidad materno-fetal tanto en países desarrollados como en subdesarrollados ${ }^{1}$. En esta revisión sistemática, Duckitt y Harrington cuantificaron el riesgo de preeclampsia asociado con los diferentes factores detectables en la primera consulta prenatal.

$\mathrm{Si}$ bien encontraron factores de riesgo asociados a preeclampsia en la primera consulta, no se pudo determinar la asociación de éstos con el inicio temprano o tardío de la enfermedad, siendo este un factor fundamental al momento de establecer pronósticos y consecuencias ocasionadas por la enfermedad ${ }^{2}$. Por otro lado, tampoco se determinó la interrelación entre los diferentes factores de riesgo: obesidad, edad materna, hipertensión crónica.

Actualmente se realizan pruebas de tamizaje para muchas enfermedades de incidencia mucho menor a la de preeclampsia (Síndrome de Down, Hepatitis B, Isoinmunización Rh, toxoplasmosis, etc.). Sin embargo, no se efectúa una identificación sistemática de mujeres en riesgo de preeclampsia. Probablemente se deba a que la preeclampsia no reúna todos los criterios necesarios para la aplicación de una prueba de tamizaje. Por ejemplo, los criterios diagnósticos no estén aún uniformemente definidos y no existe aún una intervención efectiva de prevención o terapéutica. El tamizaje de una enfermedad debe ser simple, seguro, bien tolerado, con tratamientos consecuentes costo-efectivos que brinden mejores resultados (menor morbilidad y mortalidad) que si se hubieran realizado tardíamente. Indudablemente, la preeclampsia como enfermedad carece todavía de muchos de estos atributos.

\section{Conclusiones de los comentadores}

No obstante, aunque por el momento la evaluación de riesgos para preeclampsia al comienzo del embarazo no parece tener un impacto clínico evidente, la información sobre los diferentes factores de riesgo encontradas en esta revisión sistemática puede ser de utilidad para la identificación de embarazos en riesgo y para la evaluación de futuros métodos de tamizaje, de diagnóstico y de intervenciones para la prevención o tratamiento.

Lucas Minig y Lucas Otaño [ Servicio de Obstetricia del Hospital Italiano de Buenos Aires. ]

Minig L, Otaño L. Factores de riesgo de preeclampsia identificables durante la primera consulta del control prenatal.

Evid pract ambul. 2005; 8:142. Disponible en URL: www.evidencia.org Comentado de: Kirsten Duckitt, Debora Harrington. Risk factors for preeclampsia at antenatal booking: systematic review of controlled studies. BMJ 2005;330:565-62. PMID: 15743856.

Referencias

1. Roberts JM, Pearson G, Cutler J, Lindheimer M; NHLBI Working Group on Research on Hypertension During Pregnancy. Hypertension. 2003 Mar;41(3):43745. Epub 2003 Feb 10. 